\title{
Brain Abscess after Transanal Hemorrhoidal Dearterialization: A Case Report
}

\author{
A.E.M. Berkel ${ }^{a}$ M.E. Witte ${ }^{b}$ R. Koop ${ }^{a} \quad$ M.G.R. Hendrix ${ }^{c} \quad$ J.M. Klaase ${ }^{a}$ \\ ${ }^{a}$ Department of Surgery, Medisch Spectrum Twente, Enschede, ${ }^{b}$ Department of Surgery, \\ University Medical Center Groningen, Groningen, and ' Laboratory of Microbiology Twente \\ Achterhoek, Enschede, The Netherlands
}

\section{Key Words}

Hemorrhoids · Transanal hemorrhoidal dearterialization · Brain abscess · Streptococcus milleri Septic complications

\begin{abstract}
A relatively new therapy in the treatment of hemorrhoids is transanal hemorrhoidal dearterialization (THD). We report a case of brain abscess caused by Streptococcus milleri following THD. Although a brain abscess after drainage of a perianal abscess has been described in the literature, no report exists of a brain abscess following treatment of hemorrhoids. A healthy 51-year-old man with hemorrhoids underwent THD. Two weeks later he presented with a headache, bradyphrenia, flattened behavior and a left hemiplegia. No perianal complaint and/or perianal abscess was present. A contrast CT scan of the cerebrum showed a right temporoparieto-occipital abscess, with edema and compression of the surrounding tissue and lateral ventricles. MRI showed an abscess with leakage in the right lateral ventricle. Treatment with dexamethasone and intravenous antibiotics was started. Because of progression of symptoms, 3 weeks later ventriculoscopy was performed and the abscess was drained. Culture of the punctuate showed S. milleri. Because of developing hydrocephalus 3 days after ventriculoscopy, first an external ventricle drain and later a ventriculoperitoneal drain was placed. Hereafter the hemiplegia and cognitive disorders improved. This case report describes a severe complication following treatment of hemorrhoids with THD which until now, to our knowledge, has never been described in the literature.
\end{abstract}




\section{Introduction}

Hemorrhoids are vascular cushions lining the anal canal above the dentate line [1]. When hemorrhoidal tissue gives rise to symptoms, such as bleeding, anal pruritus, soiling and prolapse, one can speak of hemorrhoidal disease. The prevalence in the western world ranges from $4.4 \%$ in adults in the United States [2] to 39\% in the adult general population in Austria [3]. Etiologic factors are multifactorial and include irregular bowel habits and prolonged straining [1].

A relatively new therapy in the treatment of hemorrhoids is hemorrhoidal artery ligation, also called transanal hemorrhoidal dearterialization (THD), where hemorrhoidal arteries, end branches of the superior rectal artery, are identified with Doppler sound and consecutively selectively ligated. This is based on the theory that hemorrhoids occur when there is an imbalance in blood flow of the hemorrhoidal plexus. By arterial ligation the inflow is reduced, causing the plexus to diminish and the hemorrhoids to shrink [1]. This therapy was introduced in 1995 by Morinaga et al. [4], and since then several studies have shown short-term results of approximately $75 \%$ success rate $[5,6]$. It is a safe procedure with only minor complications reported, such as postoperative bleeding and moderate pain, rarely constipation and perianal fissure [4-6].

In this article, we report a case of a brain abscess caused by Streptococcus milleri following THD. Severe distant septic complications after treatment of hemorrhoidal disease are reviewed.

\section{Case Report}

A 51-year-old man was seen at our outpatient clinic. He complained of soiling and a feeling of prolapse after defecation, which resolved spontaneously. His medical record consisted only of an ear operation a long time ago, he was otherwise healthy and used no medication. Proctoscopy showed internal hemorrhoids, and three rubber band ligations were placed at the left lateral, right anterior and right posterior positions. However, symptoms persisted at 2-month follow-up and a THD procedure under spinal anesthesia was carried out. Six end branches of the superior rectal artery were detected and ligated. The day care procedure was uneventful. He was seen postoperatively at 7 and 10 days on an outpatient basis. An analgesic was prescribed in order to relieve postoperative pain.

Two weeks later the patient was admitted to the Neurology Department with a headache, bradyphrenia, flattened behavior and a left hemiplegia. Laboratory tests showed a leukocyte count of $20.6 \times 10^{9} / \mathrm{l}$, a C-reactive protein of $126 \mathrm{mg} / \mathrm{l}$ and an erythrocyte sedimentation rate of $82 \mathrm{~mm} / \mathrm{h}$. A contrast CT scan of the cerebrum showed a right temporoparieto-occipital abscess (fig. 1), with edema and compression of the surrounding tissue and lateral ventricles. One day later, MRI was performed which also showed an abscess with leakage in the right lateral ventricle (fig. 2). Treatment with dexamethasone and intravenous antibiotics (penicillin two million units 6 times daily, metronidazole $500 \mathrm{mg} 3$ times daily for 2 weeks and ceftriaxone $2 \mathrm{~g}$ twice daily for 3 days) was started. Despite prolongation of antibiotic treatment, 4 days later there was progression of the bradyphrenia and the left hemiplegia. A CT scan showed reduction of the size of the abscess, but progression of ventricle size and the right frontal edema (fig. 3). There was no indication for a drain at that time. Antibiotic treatment with penicillin was continued. Because of progression of symptoms, 3 weeks later a ventriculoscopy was performed and the abscess was drained. Culture of the punctuate showed a $S$. milleri, which was sensitive to penicillin (MIC $<0.1 \mathrm{mg} / \mathrm{l}$ ). 
Cultures of blood and liquor taken at the time of ventriculoscopy remained negative. There were no perianal complaints and/or abscess formation all the time. Antibiotics were continued for 2 months. Because of developing hydrocephalus 3 days after ventriculoscopy, first an external ventricle drain and later a ventriculoperitoneal drain was placed. Hereafter the hemiplegia and cognitive disorders improved. Three months later he was discharged to a rehabilitation clinic where he stayed 2 weeks. During the rehabilitation period cognitive disorders, balance and walking function improved. Six months after discharge from the rehabilitation clinic he was back to work for $32 \mathrm{~h}$ a week.

\section{Discussion}

In this report we describe a patient who developed a brain abscess after THD, a complication that, to our best knowledge, has not been described previously. Although brain abscesses after drainage of a perianal abscess have been described in the literature [7], no report exists of brain abscesses after treatment of hemorrhoids. In our case no perianal complaint and/or abscess was present. In this case, the brain abscess caused by S. milleri developed 2 weeks after treatment of hemorrhoids by THD. THD is one of several new techniques developed to avoid the classic hemorrhoidectomy in the treatment of hemorrhoids that do not respond to conservative treatment and rubber band ligation. Until now, to our knowledge, no complications other than postoperative bleeding and moderate pain, rarely constipation and a perianal fissure have been described from THD [4-6].

Brain abscesses are localized infections of the brain substance, producing a significant degree of morbidity and mortality [8]. Infection originates via extension from adjacent foci or by metastasis through the blood stream due to episodes of bacteremia or through septic emboli from other sources of intravascular infection [8]. Both aerobic and anaerobic Streptococci account for the majority of cases of brain abscesses. $S$. milleri is the most common pathogen [8]. The S. milleri group (SMG) consists of three species (S. anginosus, S. intermedius and $S$. consellatus) and these constitute part of the normal flora of the human oral cavity, upper respiratory and gastrointestinal tract [9]. It is well recognized that SMG can cause suppurative infections, especially abscesses of the brain, spinal cord and abdominal viscera, and is quite distinct among the viridans Streptococci in this regard [9]. The predisposing factors associated with SMG infections include mucosal infection (periodontal diseases and paranasal sinusitis), pneumonia, alcohol abuse and diabetes $[8,9]$. In our patient none of these predisposing factors were present.

The incidence of transient bacteremia detected from blood cultures following digital rectal examination, proctoscopy and colonoscopy varies between 0 and 9.5\% [10]. Bacteremia following treatment of hemorrhoids has been shown to occur in $8.3 \%$ of patients following traditional hemorrhoidectomy, and 5 and $11 \%$ respectively in diathermy and stapled hemorrhoidectomy [11]. Most of these transient bacteremias are asymptomatic and do not produce metastatic disease. There is also no evidence for antibiotic prophylaxis in the treatment of hemorrhoids, except in patients with cardiac valvular disease or compromised host defense [10]. In this case, according to our national (www.swab.nl) and local guidelines, no prophylactic antibiotics were given to this low-risk patient.

Besides local septic complications following the treatment of hemorrhoids, such as perianal abscess, perineal and retroperitoneal sepsis, several distant septic complications after treatment of hemorrhoids have been reported in the literature, although relatively scarce [11]. A Bacteroides fragilis infection of a 6-year-old knee prosthesis 3 months after hemorrhoidectomy has been reported, presumably secondary to bacteremia [12]. Further- 
more at least two separate cases of meningitis have also been reported, as well as pylephlebitis and multiple cases of pyogenic liver abscesses following hemorrhoidal banding [10, 11, 13]. Another study reports a case of endocarditis leading to septic pulmonary and renal emboli following single-quadrant rubber band ligation [14]. A septic pulmonary embolus resulting in a pleural effusion, from which Gram-negative organisms were cultured, has also been reported following hemorrhoidectomy [15].

As described in the present case, a brain abscess caused by $S$. milleri is another possible severe complication of hemorrhoidal treatment, which until now has never been described in the literature.

\section{Disclosure Statement}

The authors declare that they have no conflicts of interest.

\section{References}

1 Acheson AG, Scholefield JH: Management of haemorrhoids. BMJ 2008;336:380-383.

2 Johanson JF, Sonnenberg A: The prevalence of hemorrhoids and chronic constipation. An epidemiologic study. Gastroenterology 1990;98:380-386.

3 Riss S, Weiser FA, Schwameis K, et al: The prevalence of hemorrhoids in adults. Int J Colorectal Dis 2012;27:215-220.

4 Morinaga K, Hasuda K, Ikeda T: A novel therapy for internal hemorrhoids: ligation of the hemorrhoidal artery with a newly devised instrument (Moricorn) in conjunction with a Doppler flowmeter. Am J Gastroenterol 1995;90:610-613.

5 Arnold S, Antonietti E, Rollinger G, et al: Doppler ultrasound assisted hemorrhoid artery ligation. A new therapy in symptomatic hemorrhoids. Chirurg 2002;73:269-273.

-6 Sohn N, Aronoff JS, Cohen FS, et al: Transanal hemorrhoidal dearterialization is an alternative to operative hemorrhoidectomy. Am J Surg 2001;182:515-519.

7 Doepp F, Schreiber SJ, Wandinger KP, et al: Multiple brain abscesses following surgical treatment of a perianal abscess. Clin Neurol Neurosurg 2006;108:187-190.

-8 Melo JC, Raff MJ: Brain abscess due to Streptococcus MG-intermedius (Streptococcus milleri). J Clin Microbiol 1978;7:529-532.

-9 Kowlessar PI, O'Connell NH, Mitchell RD, et al: Management of patients with Streptococcus milleri brain abscesses. J Infect 2006;52:443-450.

10 Guy RJ, Seow-Choen F: Septic complications after treatment of haemorrhoids. Br J Surg 2003;90:147-156.

11 McCloud JM, Jameson JS, Scott AN: Life-threatening sepsis following treatment for haemorrhoids: a systematic review. Colorectal Dis 2006;8:748-755.

12 Pattison RM, Cooke RP, James SE: Bacteroides fragilis infection of a knee prosthesis after haemorrhoidectomy. Lancet 1995;346:1097.

13 Chau NG, Bhatia S, Raman M: Pylephlebitis and pyogenic liver abscesses: a complication of hemorrhoidal banding. Can J Gastroenterol 2007;21:601-603.

-14 Tejirian T, Abbas MA: Bacterial endocarditis following rubber band ligation in a patient with a ventricular septal defect: report of a case and guideline analysis. Dis Colon Rectum 2006;49:1931-1933.

$\checkmark 15$ Liffmann KE, Houle DB: Septic pulmonary embolus with empyema following hemorrhoidectomy. Am J Proctol 1964;15:55-58. 
Case Reports in
Gastroenterology

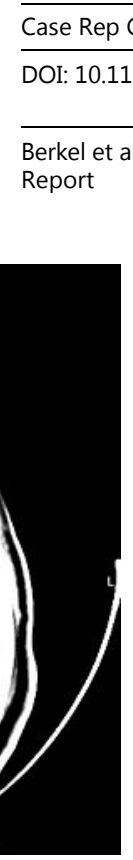

Fig. 1. CT image of the cerebrum showing a right temporoparieto-occipital abscess, with edema and compression of the surrounding tissue and lateral ventricles.

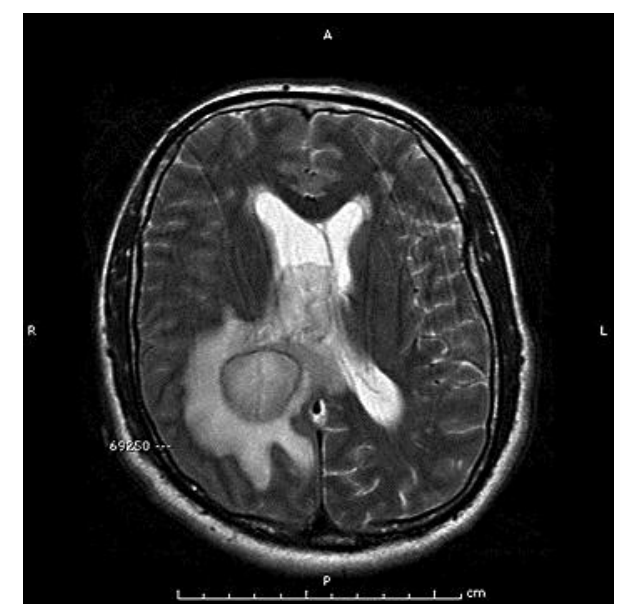

Fig. 2. One day later, T2-weighted MRI of the cerebrum with contrast showed the abscess with leakage in the right lateral ventricle. 


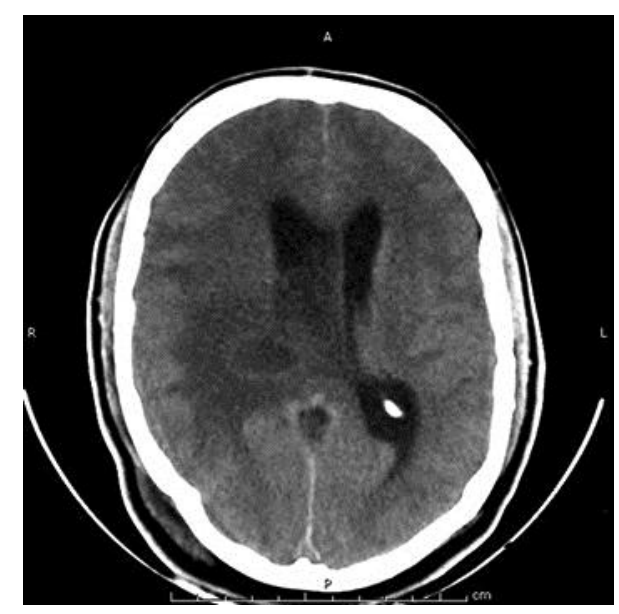

Fig. 3. Four days later, a CT image of the cerebrum showed reduction of the size of the abscess, but progression of ventricle size and the right frontal edema. 